\section{The DNAzyme anti-insulin-like growth factor-IIP3 inhibited tumour growth in a mouse model of human hepatocarcinoma}

\author{
Min Zhang ${ }^{1}$, Chun-Li Mei ${ }^{1, *}$ and Ji-Dong Liu ${ }^{2, *}$ \\ ${ }^{1}$ Medical Department, Beihua University, Jilin 132001, China \\ ${ }^{2}$ Jilin Central General Hospital, Jilin 132001, China
}

Insulin-like growth factor II promoter 3 (IGF-IIP3) is the most active promoter in live cancer cells. In this study, the anti-tumour growth effects of DRz1 were measured in a model of hepatoma-bearing mice. DRz1 significantly inhibited IGF-IIP3 expression and tumour growth in vivo. Compared to the sham-treated control group, DRz1 treatment attenuated the tumour volume and weight after 14 days of treatment. DRz1 could significantly inhibit the expression of IGF-IIP3, could inhibit the associated tumour-promoting proteins and could retard the growth of hepatocarcinoma tumours in vivo. These findings provide novel evidence for the anti-tumour effects of DRz1 in vivo.

Keywords: Anti-tumour growth, bearing mouse model, hepatocellular carcinoma, IGF-IIP3-DRz1.

HePATOCELlular carcinoma (HCC) is a major cause of morbidity and mortality worldwide, and HCC is the fifth most common cancer in males and the seventh most common cancer in females ${ }^{1}$. HCC is characterized by rapid development, early metastasis, poor prognosis and short survival time ${ }^{2}$. Traditional surgical therapies, including radiotherapy, chemotherapy, and a combination of traditional Chinese medicine and synthetic drugs, do not significantly improve the prognosis mainly because of the late presentation of large tumours; also only $10-20 \%$ of the carcinomas can be removed surgically. Even though clinical trials reported a statistically relevant increase in mean survival time, this survival comes about through the attenuation of disease progression but, ultimately does not represent a solution that leads to disease remission, which would be desirable. Following the development of genetics-based therapies for a myriad of diseases, similar treatments have been suggested for HCC based on promising preliminary results.

IGF-II is a foetal growth protein produced by liver cells. It is an important pro-angiogenic factor that can directly promote angiogenesis, and a number of liver cancer cell lines secrete it through autocrine or paracrine mechanisms ${ }^{3}$. The human $I G F-I I$ gene is a complex transcription unit containing four different promoters $(\mathrm{P} 1-\mathrm{P} 4)^{4}$. In hepatocellular carcinoma (HCC) and in cell

*For correspondence. (e-mail: 1227002496@qq.com; 477053766@qq.com) lines expressing IGF-II, P3 is the most active promoter ${ }^{5}$. P3 is also the major promoter in liver tumour tissues and in IGF-II-expressing cell lines. It is related to angiogenesis and indirectly promotes the formation of liver cancer blood vessels, thereby contributing to tumour development and invasion ${ }^{6}$.

Deoxyribozymes (DNAzymes or DRzs) are synthetic, single-stranded catalytic DNA that can be engineered to bind by Watson-Crick base pairing to complementary sequences in a target messenger RNA (mRNA); DRzs can then cleave it at predetermined phosphodiester linkages. One of the most important properties of DNAzymes is currently also one of the most actively-researched topics ${ }^{6}$. The 10-23 DRz has been proposed as a general model for $\mathrm{DRzs}^{7}$. This DRz has been widely shown (both in vitro or in vivo) to inhibit the expression of its target genes and its dependent genes ${ }^{7,8}$. Moreover, DRzs are able to attenuate the progression of a variety of pathologies in animal models, and they show significant potential as therapeutic tools $^{8}$. They can also block the development of diverse pathologies in animal models and have potential utilities as therapeutic tools.

This study is a continuation of our previous research that showed that the previously designed IGF-IIP3DNAzyme (IGF-II-DRz1) could inhibit cell proliferation and invasion and the motility and migration of SMMC7721 cells in vitro ${ }^{3,4}$. However, it is unclear whether IGFIIP3-DRz1 displays any therapeutic effects on HCC growth in intact organisms. Consequently, we tested whether IGF-IIP3-DRz1 was able to reduce IGF-II levels to such an extent that tumour growth was attenuated in a mouse model of human hepatocarcinoma. The results from the current study might be used to optimize DRzbased therapeutics for HCC and might lead to subsequent phase I clinical testing.

The IGF-IIP3-DNAzyme (DRz1) ${ }^{4}$ was designed as previously described by Dass et al. ${ }^{9}$. Briefly, a region of 10-23 DRz was made by incorporating two $8 \mathrm{bp}$ arms complementary to the target gene into each side of the catalytic motif. As a control, an inactive, sequenceinverted DNAzyme (DRz1 ${ }^{\text {inv }}$ ) was designed based on the flipped sequence of DRz1. Both DNAzymes were synthesized by Shanghai Sangon Biotechnology Co. Ltd (Shanghai, China). The sequences of human IGF-IIP3 mRNA templates and the corresponding DNAzymes were designed as follows (arrow indicates the cleavage point):

Template-1: 5' AGACACCAA $\downarrow$ UGGGAAUC 3'

DNAzyme 1 (DRz1): 5' GATTCCCAGGCTAGCTACAACGATGGTGTCT 3'

Inactive sequence:

Inverted DNAzyme (DRz1 $\left.{ }^{\text {inv }}\right)$ : 5' TCTGTGGTAGCAACATCGATCGGACCCTTAG 3'

In this study, the SMMC-7721 cell line was used as an HCC source and was purchased from the Cell Bank and 
Type Culture Collection of the Chinese Academy of Sciences (Shanghai Institute of Cell Biology, Chinese Academy of Sciences, Shanghai, China). The cells were maintained and cultured in Dulbecco's Modified Eagle Medium (DMEM) supplemented with 10\% foetal bovine serum (FBS; Gibco/Life Technologies, Grand Island, NY, USA), $100 \mathrm{IU} / \mathrm{ml}$ penicillin, $100 \mathrm{IU} / \mathrm{ml}$ streptomycin and $2 \mathrm{mmol} / \mathrm{l} \mathrm{L}$-glutamine in a humidified atmosphere $\left(5 \% \mathrm{CO}_{2}, 37^{\circ} \mathrm{C}\right)$. The cells in the suspensions were counted using a Cedex analyzer (Innovatis AG, Bielefeld, Germany).

Twenty-four healthy male nude mice (weight 18-20 g, 4-5 weeks-old) were obtained from the Beijing Laboratory Animal Centre (Chinese Academy of Sciences, Beijing, China. The mice were housed in a pathogen-free laboratory animal centre, maintained at $\sim 22^{\circ} \mathrm{C}$ under a $12 \mathrm{~h}$ dark/light cycle and given food and water ad libitum. All experimental procedures that involved lab animals were performed according to the Institutional Animal Care and Use Committee's guidelines of Beihua University and were approved by the Experimental Animal Ethics Committee of Beihua University.

Briefly, $5 \times 10^{6}$ SMMC-7721 cells were suspended in $200 \mu \mathrm{l}$ PBS and were inoculated subcutaneously into nude mice to set up the SMMC-7721 tumour-bearing animal model. After 4 weeks, when the tumours were approximately $4-5 \mathrm{~mm}$ in diameter (measured with a caliper), the mice were randomly divided into three groups of eight mice each: (i) a sham-treated control group, (ii) an inactive $\mathrm{DRz}$ group (DRzl ${ }^{\text {inv }}$ ) and (iii) a DRz1 group. Each mouse received daily, multipoint intratumoural injections with $10 \mu \mathrm{g}$ DRz1 or DRz1 ${ }^{\text {inv }}$ dissolved in $10 \mu \mathrm{l}$ PBS. The control group received an equal volume of PBS. The tumour sizes were monitored every two days, and the tumour volume was calculated using the following formula: $V=\left(\right.$ length $\left.\times w_{\text {idth }}^{2}\right) / 2$. After 14 days of daily injections, the mice in each group were sacrificed by decapitation, and the tumours were excised. Postmortem, the body weight of the mice, the excised tumour dimensions and the excised tumour wet weight were determined. Subsequently, the tumours were either flash frozen in liquid nitrogen or were fixed in $4 \%$ paraformaldehyde overnight, followed by cryoprotection in $25 \%$ sucrose for several days. The cryoprotected tumours were then washed with $0.1 \%$ PBS prior to being embedded in optimal cutting temperature (OCT) compound; the tumours were then sectioned into 8 micron sections. For analysis of the mRNA and protein expression levels in tumours, the OCT compound-embedded tumour sections were scraped from glass slides containing individual tumours; the scraped tumours were pooled according to the treatment group to obtain sufficient tissue for further analysis. The tissues were frozen in liquid nitrogen and were stored at $-80^{\circ} \mathrm{C}$ until analysis.

The IGF-IIP3 and VEGF mRNA expression levels in the excised tumour tissue of the various treatment groups were quantified by real-time PCR. The housekeeping gene $\beta$-actin was used as a control. Human IGF-II, VEGF and $\beta$-actin PCR primers were designed using Primer Express software (Perkin-Elmer Biosystems, USA) based on their published sequences (Table 1). The total RNA was isolated from the frozen tumour tissue with TRIzol Reagent (Invitrogen, Rockville, MD, USA) according to the manufacturer's instructions. To avoid DNA contamination, the total RNA was treated with RNase-free DNase I (Takara, Kyoto, Japan) for $60 \mathrm{~min}$ at $37^{\circ} \mathrm{C}$ and was extracted once more with the TRIzol reagent. The RNA purity was determined from the $260 / 280 \mathrm{~nm}$ absorbance ratio, and the RNA integrity was assessed by determining the intensity of the $28 \mathrm{~S}$ and $18 \mathrm{~S}$ rRNA bands after formaldehyde agarose gel electrophoresis. The total RNA $(2 \mu \mathrm{g})$ was subjected to reverse transcription using a RevertAid $^{\mathrm{TM}}$ First-Strand cDNA Synthesis Kit (Fermentas Inc., Glen Burnie, MD, USA) with a random hexamer primer; cDNA solution $(2 \mu \mathrm{l})$ was used for real-time PCR. The genes were amplified in a $25 \mu$ reaction volume using SYBR Universal PCR Master Mix (Tiangen Biotech, Beijing, China) on a MiniOpticon ${ }^{\mathrm{TM}}$ Real-time PCR System (Bio-Rad, Hercules, CA, USA). The temperature profile consisted of an initial denaturation step at $95^{\circ} \mathrm{C}$ for $5 \mathrm{~min}$, followed by 40 cycles of $95^{\circ} \mathrm{C}$ for $10 \mathrm{sec}$, $58^{\circ} \mathrm{C}$ for $15 \mathrm{sec}$ and $72^{\circ} \mathrm{C}$ for $10 \mathrm{sec}$ before being subjected to a melting curve analysis ${ }^{10}$. The specificity of the amplified products was evaluated via agarose gel electrophoresis and was further verified with automated cycle sequencing. To ensure consistency in the threshold cycle $(C t)$ values, triplicate reactions were performed, and the mean $C t$ values were used for calculating the relative expression levels. The $C t$ values were analysed as described previously by Zhou et al. ${ }^{11}$, and the normalized $C t$ values for each gene were subjected to Student's $t$-test with a two-tailed distribution to determine the statistical significance $(95 \%$ confidence interval). The final measurements were carried out in triplicate, and the mean values were calculated. For standardization purposes, $\beta$-actin was used as an internal control in each sample.

The IGF-IIP3, VEGF, caspase-3 and caspase-9 protein expression levels from the frozen tumour tissues of various treatment groups were quantified by western blot. The tumour tissues were cut into small pieces, weighed and washed twice with pre-cooled PBS. The tumour

Table 1. Oligonucleotide sequences and the product sizes of primers

\begin{tabular}{lcc}
\hline cDNA & Size (bp) & \multicolumn{1}{c}{ Oligonucleotide primers } \\
\hline IGF-IIP3 & \multirow{2}{*}{381} & F: 5'-ATGGGAATGCCAATGGGGAAG-3' \\
& & R: 5'-CTTGCCCACGGGGTATCTGGG-3' \\
& & F: 5'-TACCTCCACCATGCCAAGTGGT-3' \\
VEGF & 435 & R: 5'-AGGACGGCTTGAAGATGTAC-3' \\
& & F: 5'-ATCATGTTTGAGACCTTCAACA-3' \\
$\beta$-Actin & 318 & R: 5'-CATCTCTTGCTCGAAGTCCA-3'
\end{tabular}


tissue was treated with RIPA buffer $(50 \mathrm{mmol} / \mathrm{l}$ Tris $(\mathrm{pH}$ 8.0), $150 \mathrm{mmol} / \mathrm{l} \mathrm{NaCl}, 0.1 \%$ SDS, $1 \% \mathrm{NP} 40$ and $0.5 \%$ sodium deoxycholate) containing protease inhibitors $(1 \%$ cocktail and $1 \mathrm{mmol} / \mathrm{l} \mathrm{PMSF})$. The tumour tissue was homogenized with a low-speed homogenizer for $30 \mathrm{sec}$ after each time interval of $1 \mathrm{~min}$ in an ice bath until lysis was complete. The lysate was centrifuged at $14,000 \mathrm{~g}$ with an ice-cold centrifuge for $15 \mathrm{~min}$. The supernatant was immediately transferred and saved at $-80^{\circ} \mathrm{C}$ until analysis. The total protein extracts were separated on a $15 \%$ SDS-PAGE and were transferred to PVDF membranes. The membranes were blocked with tris-buffered saline containing $0.1 \%$ Tween 20 (pH 7.6, [TBS-T]) for $1 \mathrm{~h}$ at room temperature. Subsequently, the PVDF membranes were immunoblotted overnight at $4{ }^{\circ} \mathrm{C}$ with the first antibody (see below) solution (1: 1000). After washing twice with TBS-T, the membrane was incubated with an HRP-labelled secondary goat anti-mouse $\operatorname{IgG}_{2 \mathrm{a}}$-B antibody (SC-2073) for $1 \mathrm{~h}$ at room temperature and was washed three times with TBS-T. The final detection was performed with enhanced chemiluminescence (ECL) western blotting reagents (GE Healthcare, Piscataway, NJ, USA), and the membranes were exposed to the LumiFilm Chemiluminescent Detection Film (Roche Applied Science, Rotkreuz, Switzerland).

The loading differences were normalized by using a monoclonal $\beta$-actin antibody against the housekeeping control $\beta$-actin. The primary antibodies used in this study included the following: anti-IGF-IIP3 (SC-7435), anticaspase-3 (SC-7272), VEGF (SC-7269) and anti- $\beta$-actin (SC-130301) and were all acquired from Santa Cruz Biotechnology, Inc. (Santa Cruz, CA, USA).

The tumours were immersed in $4 \%$ paraformaldehyde for $4 \mathrm{~h}$ and were transferred to $70 \%$ ethanol. Individual lobes of tumour biopsy material were placed in processing cassettes, dehydrated through a serial alcohol gradient, and embedded in paraffin wax blocks. Before immunostaining, $5 \mu \mathrm{m}$-thick lung tissue sections were dewaxed in xylene, rehydrated with decreasing concentrations of ethanol and washed in PBS. The cells were then stained with hematoxylin and eosin (H\&E). After staining, the sections were dehydrated through increasing concentrations of ethanol and xylene ${ }^{12}$.

The measurements were performed in triplicate, and the results expressed as the means $\pm \mathrm{SD}$. One-way analysis of variance (ANOVA) or Student's $t$-tests were carried out using statistical analysis software (SPSS, Chicago, IL, USA). The tumour growth over time was analysed among the three groups by two-way ANOVA using Graphpad Prism version 4.0b software (Graphpad Software, Inc., La Jolla, CA, USA). The values of $P<0.05$ were considered to be statistically significant.

DRz1 was able to attenuate the IGF-IIP3 mRNA and protein expression concomitantly by nearly $50 \%$ in SMMC-7721 cells ${ }^{4}$. This downregulation of IGF-IIP3 expression was associated with increased caspase- 3 and caspase- 9 activity and inhibited the cell proliferation and increased apoptosis. We evaluated the effectiveness of DRzl in human hepatocarcinoma tumours grafted subcutaneously onto nude mice. The grafted tumours were treated daily with $1 \mu \mathrm{g} / \mu \mathrm{l}(10 \mu \mathrm{g} / 10 \mu \mathrm{l}$ PBS $)$ DRz1, $\mathrm{DRz}^{\text {inv }}$ or $10 \mu \mathrm{l}$ PBS alone for 14 days via multipoint intra-tumoural injections.

Subjectively, none of the animals showed any signs of distress or disruption to their feeding or sleep cycles. Furthermore, the results in Figure 1 show no significant differences in body weight development; therefore, the discomfort or disruption of bodily homeostasis due to tumour growth may have been minimal. During excision, the capillaries were observed visually in the vicinity of the tumour grafts, and several sprouts penetrated the excised tumours (Figure $2 b$ ).

As shown in Figure 2, compared to the sham-treated control group, DRz1 treatment attenuated the tumour volume and weight after 14 days of treatment by a factor of 1.40 and 1.41 respectively (Figure $2 a$ ). Furthermore, the appropriateness of the chosen negative control, $D R z 1^{\text {inv }}$, is reflected in the fact that no significant differences were observed between the sham-treated and DRz $1^{\text {inv }}$ groups.

Our results show that IGF-IIP3-DRz1 could inhibit the IGF-IIP3 mRNA and protein levels in tumours and that DRzl could inhibit tumour growth without obvious adverse effects. This anti-tumour growth effect was related to the inhibition of IGF-II expression by DRz1. Similar anti-tumour growth effects were reported in DZ2, a DNAzyme that targets aurora kinase A, induces apoptosis and inhibits tumour growth in human prostate cancer ${ }^{7}$.

To evaluate to what extent DRz1 was able to attenuate the effects of IGF-II expression in the solid tumour mass, the IGF-IIP3 mRNA and protein levels were determined. Our results show that the mRNA levels were effectively

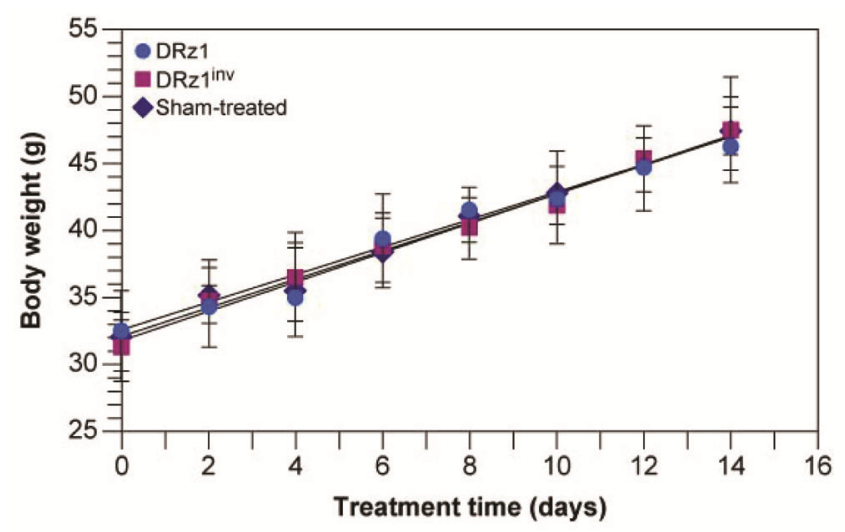

Figure 1. Effect of anti-IGF-II DNAzyme treatment on the body weight of xenografted hepatocarcinoma tumour-bearing nude mice. Body weight was assessed every 2 days, and the concomitant tumour size was evaluated. The data are presented as the means \pm SD. DRz1 ${ }^{\text {inv }}$ : Inactive, inverted DNAzyme sequence; IGF-II: Insulin-like growth factor II. 
attenuated by approximately $1.49-66.9 \pm 5.7 \%$ of shamtreated control within cells from tumour cross-sections treated consecutively with $10 \mu \mathrm{g}$ DRzl (Figure $3 a$ ). These results show the basic capacity of DRzl to attenuate $I G F-I I P 3$ gene expression. Equal protein expression was attenuated by approximately $1.47-68 \pm 4.9 \%$ of the control (Figure $3 \mathrm{~b}$ ). Furthermore, no additional effect was effectively observed at concentrations above $10 \mu \mathrm{g}$ DRz1, as the mRNA and protein expression levels levelled off to a plateau. The sequence-inverted control $\mathrm{DRz}$ did not affect the IGF-IIP3 expression levels. It is likely that, at least partially, the degradation of the DRzs by tumour-endogenous exonucleases abolishes the knockdown of IGF-IIP3 expression and leads to the restoration of IGF-IIP3 expression over time. The 10-23 DNAzymes targeting GATA-3 mRNA have recently been developed, and their anti-asthmatic effect in mouse models has been successfully demonstrated ${ }^{13}$; DNAzymes have been widely applied as a new interference strategy in the treatment of cancer ${ }^{14}$.

Vascular endothelial growth factor (VEGF) is one of the hallmarks of malignant tumour formation, invasiveness and growth, and we previously showed that DRz1 was able to inhibit VEGF in cultures of SMMC-7721
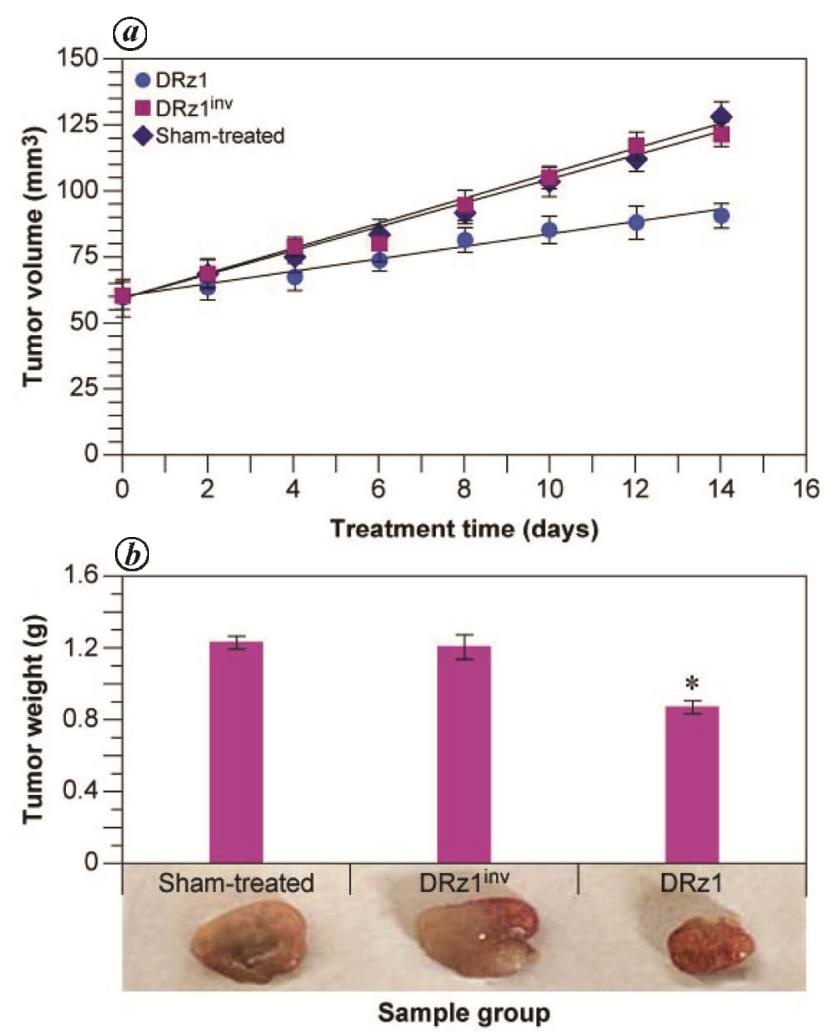

Figure 2. Effect of DRzl on xenograft hepatocarcinoma tumours in nude mice. $\boldsymbol{a}$, Tumour volume was assessed with a caliper every 2 days just before the DRz injection. $\boldsymbol{b}$, Mean tumour weight immediately after resection and after 14 days of DRz therapy. Data are presented as the means \pm SD. $* P<0.05$ compared to the sham-treated PBS group or the DRz1 ${ }^{\text {inv }}$ group. DRz1 ${ }^{\text {inv }}$ : Inactive, inverted DNAzyme sequence; IGF-II: Insulin-like growth factor II. cells ${ }^{3}$. Therefore, we assayed the expression of VEGF in response to treatment with DRz1 via real-time PCR and Western blotting to evaluate the capacity of DRz1 to inhibit VEGF in the tumour and paratumour tissues. The results in Figure 4 show that DRz1 was able to significantly downregulate the expression of VEGF in both the tumour and paratumoural tissues, whereas no difference was observed in the sequence-inverted and sham-treated controls. The magnitude of down-regulation was equally large in the tumour (approximately 1.53-65.4 $\pm 7.2 \%$ ) and in the surrounding tissues (approximately 1.47$67.9 \pm 4.7 \%$ ). Immunohistochemical techniques have been used to evaluate the expression of VEGF in tumours.

VEGF is a major regulator of the blood vessel growth that is required for tissue differentiation and function. Remarkably, the aberrant expression of VEGF in tumours and in paratumours is a hallmark of malignant tumour development. Previous findings indicate that DRz1 inhibits
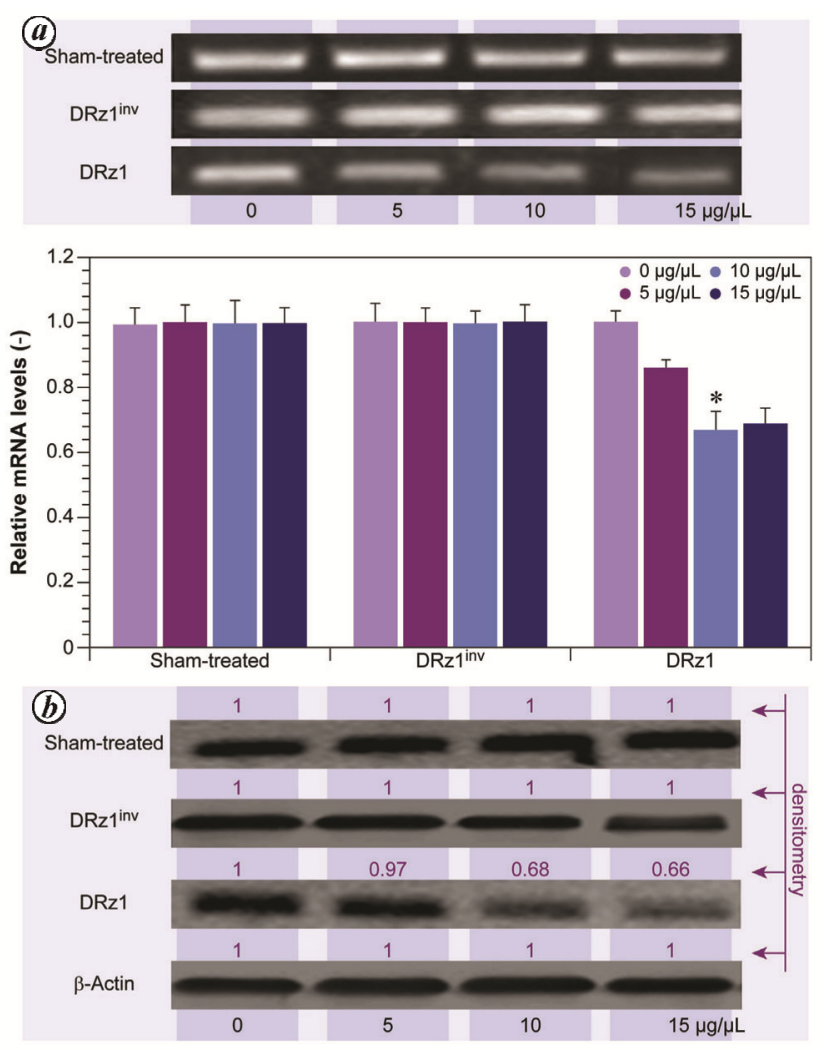

Figure 3. Effect of the anti-IGF-II DNAzyme DRz1 on insulin-like growth factor IIP3 (IGF-IIP3) expression in xenografted hepatocarcinoma tumours in nude mice. $\boldsymbol{a}$, IGF-IIP3 mRNA expression after 14 days of treatment; top: representative blot. Data shown are the means \pm SD of normalized, relative IGF-IIP3 mRNA levels $(n \geq 3)$. *Denotes a significant difference from the sham-treated control $(P<0.05)$. b , Representative western blots of IGF-IIP3 protein levels. Protein expression was quantified via densitometry and was compared with the sham-treated control. The maximal grey value (black) was set to 1 , and all other results are expressed relative to this value $(n \geq 3)$. All gels and blots were run under the same conditions. DRz $1^{\text {inv }}$ : Inactive, inverted DNAzyme sequence. 


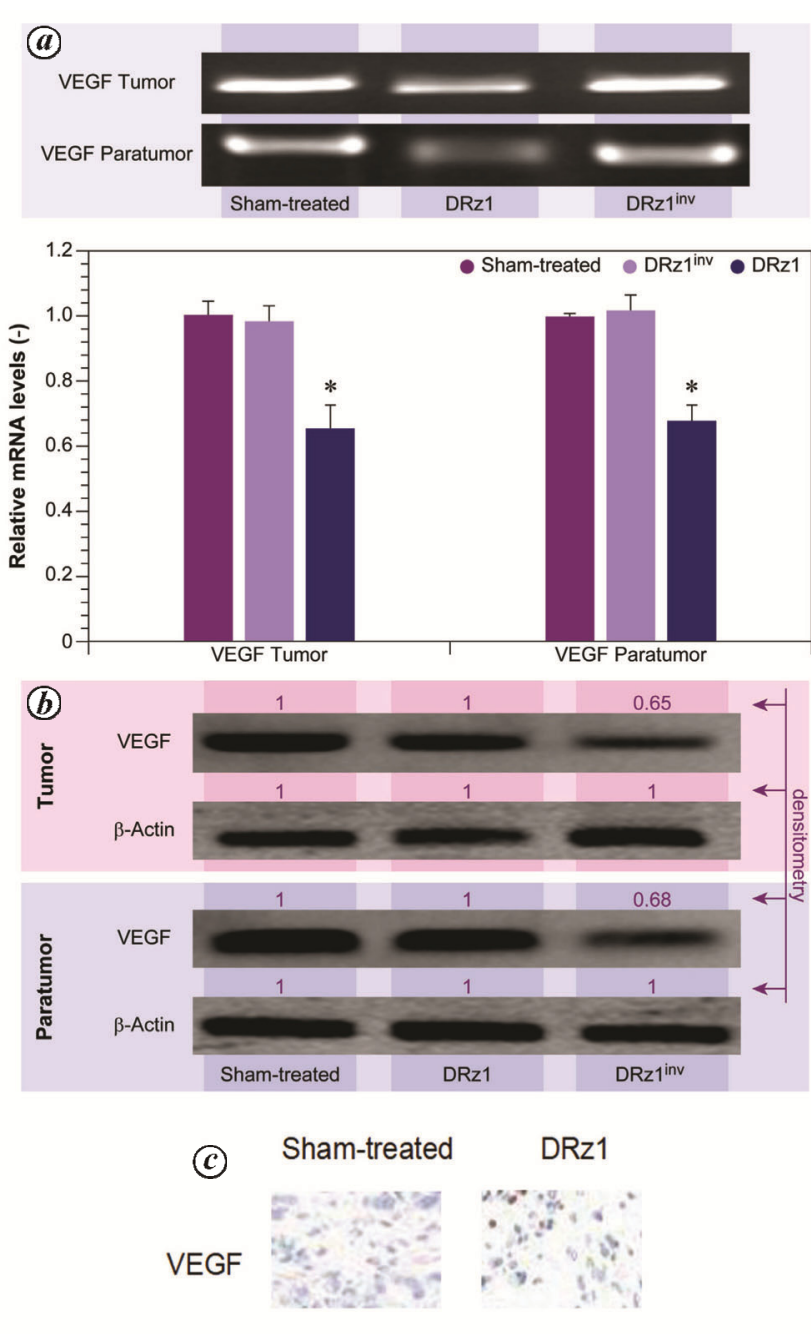

Figure 4. Effect of the anti-IGF-II DNAzyme DRz1 $(10 \mu \mathrm{g} / \mu \mathrm{L})$ on vascular endothelial growth factor (VEGF) expression in xenografted hepatocarcinoma tumours in nude mice. $\boldsymbol{a}$, VEGF mRNA expression after 14 days of treatment; top: representative blot. Data shown are the means $\pm \mathrm{SD}$ of normalized, relative VEGF mRNA levels $(n \geq 3)$ *Denotes a significant difference from the sham-treated control $(P<0.05)$. b, Representative Western blots of VEGF protein levels. Protein expression was quantified via densitometry and was compared with the sham-treated control. The maximal grey value (black) was set to 1 , and all other results are expressed relative to this value $(n \geq 3)$. All gels and blots were run under the same conditions. DRz $1^{\text {inv }}$ : Inactive, inverted DNAzyme sequence; IGF-II: Insulin-like growth factor II. $c$, Immunohistochemical assay of VEGF in the tumour tissues.

cell migration in SMMC-7721 cells ${ }^{3}$, and DRzs markedly decrease VEGF expression. In addition, DRzs significantly suppress the expression of MMP-9, but not of MMP-2, in SMMC-7721 cells. MMPs are proteolytic enzymes that degrade the extracellular matrix and the basement membranes of cells, and MMP-2 and MMP-9 are the most vital enzymes for degradation ${ }^{15}$. Therefore, they are considered to have major significance for tumour invasion and metastasis. Our studies have previously demonstrated that DRzs could inhibit the invasion and motility of SMMC-7721 cells by a mechanism that involves the downregulation of MMP-9 and VEGF. In this study, our

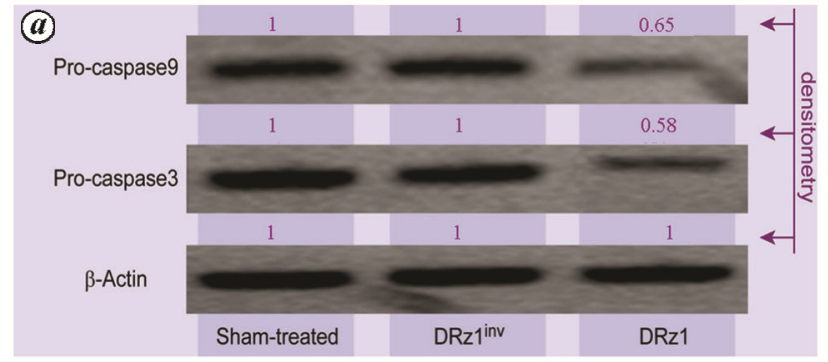

(b) Sham-treated

DRz1

Pro-caspases 3

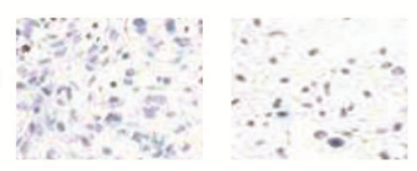

Figure 5. Effect of the anti-IGF-II DNAzyme DRz1 $(10 \mu \mathrm{g} / \mu 1)$ on the activation of caspases in xenografted hepatocarcinoma tumours in nude mice. $\boldsymbol{a}$, Representative Western blots of key pro-caspase protein levels after 14 days of treatment. Protein expression was quantified via densitometry and was compared with the sham-treated control. The maximal grey value (black) was set to 1 , and all other results were expressed relative to this value $(n \geq 3)$. All gels and blots were run under the same conditions. $\boldsymbol{b}$, Immunohistochemical assay of VEGF in the tumour tissues. DRz1 ${ }^{\text {inv }}$ : Inactive, inverted DNAzyme sequence; IGF-II: Insulin-like growth factor II.

results show that DRzl could reduce the expression of VEGF in tumour and paratumour tissues (Figure 3) and could inhibit migration in vivo.

To evaluate the extent that DRzl was able to activate the caspase-3/9 pathway in the solid tumour tissue, the procaspase- 3 and 9 expression levels were determined in the tumour tissue. As shown in Figure 5, compared to the expression levels, the control group (the PBS injection group), the procaspase-3 and procaspase- 9 expression levels decreased significantly in the DRz1-treated group, whereas inactive DRzl showed no effect on the expression levels of procaspase-3 and procaspase-9.

Our results generally demonstrate that DRz1 could significantly inhibit the expression of IGF-IIP3 and the associated tumour-promoting proteins, and DRz1 could retard the growth of hepatocarcinoma tumours in vivo. These findings provide proof-of-principle results and are the basis for the further development of this DNAzyme as a novel therapeutic intervention for liver cancer treatment, although further development is required.

Conflict of interest: The authors declare no conflicts of interest.

1. Chen, P. J. et al., Issues and controversies of hepatocellular carcinoma-targeted therapy clinical trials in Asia: experts' opinion. Liver Int., 2010, 30(10), 1427-1438.

2. El-Serag, H. B., Davila, J. A., Petersen, N. J. and McGlynn, K. A., The continuing increase in the incidence of hepatocellular carcinoma in the United States: an update. Ann. Intern. Med., 2003, 139(10), 817-823. 
3. Zhang, M., Zhao, H., Luo, F., Luo, S. and Shi, W., IGF-II inhibitory DNAzymes inhibit the invasion and migration of hepatocarcinoma cells. Biotechnol. Lett., 2011, 33(5), 911-917.

4. Zhang, M., Drummen, G. P. and Luo, S., Anti-insulin-like growth factor-IIP3 DNAzymes inhibit cell proliferation and induce caspase-dependent apoptosis in human hepatocarcinoma cell lines. Drug Des. Dev. Ther., 2013, 7, 1089-1102.

5. Breuhahn, K. and Schirmacher, P., Reactivation of the insulin-like growth factor-II signaling pathway in human hepatocellular carcinoma. World J. Gastroenterol., 2008, 14(11), 1690-1698.

6. Dass, C. R., Saravolac, E. G., Li, Y. and Sun, L. Q., Cellular uptake, distribution, and stability of 10-23 deoxyribozymes. Antisense Nucleic Acid Drug Dev., 2002, 12(5), 289-299.

7. Qu, Y. et al., Effects of DNAzymes targeting Aurora kinase A on the growth of human prostate cancer. Cancer Gene Ther., 2008, 15(8), 517-525

8. Niewiarowska, J., Sacewicz, I., Wiktorska, M., Wysocki, T., Stasikowska, O., Wagrowska-Danilewicz, M. and Cierniewski, C. S., DNAzymes to mouse beta1 integrin mRNA in vivo: targeting the tumor vasculature and retarding cancer growth. Cancer Gene Ther., 2009, 16(9), 713-722.

9. Dass, C. R., Galloway, S. J. and Choong, P. F., Dz13, a c-jun DNAzyme, is a potent inducer of caspase-2 activation. Oligonucleotides, 2010, 20(3), 137-146.

10. Benard, V., Bohl, B. P. and Bokoch, G. M., Characterization of rac and cdc42 activation in chemoattractant-stimulated human neutrophils using a novel assay for active GTPases. J. Biol. Chem., 1999, 274(19), 13198-13204.

11. Zhou, H. et al., RNAi targeting urokinase-type plasminogen activator receptor inhibits metastasis and progression of oral squamous cell carcinoma in vivo. Int. J. Cancer, 2009, 125(2), $453-462$.

12. Abellan, R. et al., Immunoassays for the measurement of IGF-II, IGFBP-2 and -3 , and ICTP as indirect biomarkers of recombinant human growth hormone misuse in sport. Values in selected population of athletes. J. Pharm. Biomed. Anal., 2008, 48(3), 844852.

13. Sel, M. et al., Effective prevention and therapy of experimental allergic asthma using a GATA-3-specific DNAzyme. J. Allergy Clin. Immunol., 2008, 121, 910-916.

14. Schubert, S., Gul, D. C., Grunert, H. P., Zeichhardt, H., Erdmann, V. A. and Kurreck, J., RNA cleaving '10-23' DNAzymes with enhanced stability and activity. Nucleic Acids Res., 2003, 31(20), 5982-5992.

15. Hallett, M. A., Teng, B., Hasegawa, H., Schwab, L. P., Seagroves, T. N. and Pourmotabbed, T., Anti-matrix metalloproteinase-9 DNAzyme decreases tumor growth in the MMTV-PyMT mouse model of breast cancer. Breast Cancer Res., 2013, 15(1), R12.

ACKNOWLEDGEMENTS. This work was partially supported by the Health Department of Jilin Province (No. 2013ZC029, Chun Li Mei and Min Zhang); the Health Department of Jilin Province (No. 2014Q003, Liu Ji dong and Min Zhang); the National Natural Science Foundation of China (No. 81541168, Min Zhang and Chun Li Mei); and the Education Department of Jilin Province in China (No. 2015153, Chun Li Mei and Min Zhang).

Received 27 March 2018; revised accepted 1 March 2019

doi: $10.18520 / \mathrm{cs} / \mathrm{v} 116 / \mathrm{i} 12 / 2072-2077$

\section{Fluoride removal by novel composite material and its performance in the fixed-bed column filter}

\author{
Mahipal ${ }^{1}$, Hasan Ahmed Faisal ${ }^{1}$, Arkamitra Kar ${ }^{2}$ \\ and Trishikhi Raychoudhury ${ }^{1, *}$ \\ ${ }^{1}$ Department of Civil and Environmental Engineering, \\ Indian Institute of Technology Patna, Bihta, Patna 801 103, India \\ ${ }^{2}$ Department of Civil Engineering, BITS-Pilani Hyderabad Campus, \\ Jawahar Nagar, Hyderabad 500 078, India
}

The objectives of this study are to (i) evaluate the process controlling fluoride $\left(\mathrm{F}^{-}\right)$removal by ceriumimpregnated activated carbon (AC-Ce) composite, and (ii) assess the performance of that composite in the fixed-bed column as a filter. The AC-Ce composites were synthesized by collecting $\mathrm{AC}$ from different sources $\left(A C_{\text {Darco }}\right.$ and $\left.A C_{E c o}\right)$. The observations from this study suggest that the homogeneous distribution of amorphous Ce within $\mathrm{AC}_{\text {Darco }}-\mathrm{Ce}$ results in high $\mathrm{F}^{-}$ sorption. Formation of crystalline $\mathrm{CeO}_{2}$ and small pore size of $\mathrm{AC}_{\mathrm{Eco}}$ inhibits $\mathrm{F}^{-}$sorption by $\mathrm{AC}_{\mathrm{Eco}}-\mathrm{Ce}$. The $\mathrm{AC}_{\text {Darco }}-\mathrm{Ce}$ composite in fixed-bed filter media can treat up to $722 \mathrm{ml}$ of $\mathrm{F}^{-}$contaminated water.

Keywords: Activated carbon, cerium, composite material, fixed-bed column, fluoride removal.

FLUORIDE $\left(\mathrm{F}^{-}\right)$contamination in groundwater is a major problem in many parts of the world. The accepted limit of $\mathrm{F}^{-}$concentration in drinking water is $1.5 \mathrm{mg} / \mathrm{l}$. Aluminabased adsorbent and precipitate are commonly used for defluoridation of water. However, leaching of aluminium from the adsorbent is a major concern for its application ${ }^{1}$. In recent years, several studies have focused on developing novel composites and nanomaterials as adsorbents for defluoridation of water. For example, zirconiumimpregnated collagen fibre ${ }^{2}, \mathrm{Mg}-\mathrm{Al}-\mathrm{Zr}$ triple-metal composite $^{3}$, metal-impregnated granular activated carbon $(\mathrm{AC})^{4-6}$, alum-impregnated activated alumina ${ }^{7}$ are synthesized and applied for removal of $\mathrm{F}^{-}$from water. Several recent studies have demonstrated that cerium $(\mathrm{Ce})$, either used in nano-particulate or composite form, exhibits excellent performance in removing $\mathrm{F}^{-}(4.37-153 \mathrm{mg} / \mathrm{g})^{4,7-9}$. Granular AC is also applied widely as a filtering material for water purification, due to its high specific surface area (SSA) and good mechanical resistance. The surface of carbon granule can be modified with metal-based functional groups, which have a strong affinity towards $\mathrm{F}^{-}$ (ref. 1). This modification of AC with metal not only improves the $\mathrm{F}^{-}$removal efficiency, but due to its high mechanical resistance the composite can also be used directly as filter medium in a fixed-bed column. One of

*For correspondence. (e-mail: trishikhi@iitp.ac.in) 